\title{
Telephone follow-up does not significantly improve glycaemic control in type 2 diabetes overall, but more intensive programmes may have an effect
}

\author{
Holly Blake \\ University of Nottingham, Nottingham, UK.
}

Commentary on: Wu L, Forbes A, Griffiths P, et al. Telephone follow-up to improve glycaemic control in patients with type 2 diabetes: systematic review and meta-analysis of controlled trials. Diabet Med 2010;27:1217-25.

\section{Implications for practice and research}

Implications for nursing practice

- Telephone follow-up is more effective at improving glycaemic control if it includes a more interactive element based on the level of clinical need.

- Such models could be important in helping to manage the increasing demand for diabetes care.

Implications for nursing research

- Research is needed which will help to determine the added value of the psychological element of the intervention (eg, motivational interviewing (MI)).

- Understanding more about the potential influence of call provider skill and expertise (professional vs nonprofessional) is needed to determine the optimum level of training required.

- Cost-benefit analysis is recommended.

\section{Context}

Diabetes is becoming increasingly common worldwide, representing a huge public health problem. In the UK, diabetes is thought to affect approximately 2.8 million people overall, and it is estimated that there may be a further 850000 
people with undiagnosed diabetes. Over $90 \%$ of people with diabetes have type 2 diabetes (T2DM), and there are an increasing number of younger adults with T2DM than previously, with the condition now developing also in children. T2DM is complex in causation and complicated to manage since it is associated with significant complications affecting the eye, the nervous system and the kidney. There is a substantial increase in risk of cardiovascular disease and a significant reduction in life expectancy. Achieving optimal glycaemic control is a key clinical objective in reducing complications, and this relies heavily on patient's adherence to medical advice on self-care regimes. Patient education programmes incorporating psychological techniques are used to support patients in translating self-care advice into self-care behaviours, such as dietary and exercise regimes, taking medication and monitoring blood glucose levels. Ongoing support is crucial in adjusting care and reinforcing positive selfmanagement behaviours.

Identifying effective systems of support represents a significant challenge, although developments in innovative new technologies have led to an increase in the use of telemedicine in clinical care, to provide ongoing support while reducing the need for costly clinic visits. Telemedicine generally refers to the use of communications and information technologies for the delivery of clinical care, and it ranges from the simple discussion of a case over the telephone to satellite technology for real-time consultations, and internet, email or mobile phone text messaging for ongoing support and follow-up with patients. In healthcare, the capabilities and accessibility of new technologies have been recognised in preventing, diagnosing, monitoring and treating disease and the development and delivery of preventive measures and proactive health promotion programmes. Telephone support is recognised as a widely accessible and low cost medium for the provision of ongoing support in clinical care, which aims to reduce the need for unnecessary clinical consultations. This medium has shown to be acceptable to patients with T2DM, ${ }^{1}$ is frequently utilised in clinical practice as a method of supporting self-care behaviours and has the potential to address a variety of chronic illnesses that can be effectively treated or prevented via the modification of lifestyle behaviours. 
There is a growing evidence base surrounding the effects of telephone follow-up in diabetes management, and given the increasing interest and application of this technology in clinical provision, a systematic review and meta-analysis was warranted.

\section{Methods}

This article sought to examine the impact of telephone follow-up interventions on glycaemic control in patients with T2DM. The authors conducted a systematic review and meta-analysis of randomised controlled trials which examined the effect of a telephone follow-up intervention on HbA1c levels. A rigorous review protocol was adopted which had been approved by the Cochrane Collaboration Metabolic and Endocrine Disorders. Electronic searches were screened by two authors and validated by a third; 8389 studies were screened, 36 selected for full assessment to the review inclusion criteria and 7 studies were included in the review, with sample sizes ranging from 62 to 608 (data available on a total of 1764 patients). Data were extracted on study design, characteristics of patients, exact nature of the telephone intervention and details of comparison. Included trials were subjected to critical appraisal to establish potential bias in selection, attrition and detection, following which pooled standardised effects were calculated for the primary outcome following Cochrane guidelines.

\section{Findings}

Despite limitations of the review, the authors highlight some key findings which may be important in helping to manage the increasing demand for diabetes care. A high level of heterogeneity was evident in the interaction within the telephone follow-up interventions. Findings demonstrated that overall, telephone followup had a limited impact on glycaemic control, but the authors concluded that follow-up was more effective if it included intensive support with a more interactive element based on clinical need (individualised targeted approach). This is consistent with interactive behaviour change technologies which highlight the need for tailored interventions based on patient need, ${ }^{2}$ and other studies reporting that the dynamics of interactions supporting the delivery of 
telephone counselling is important in that telephone advice must include personalised and/or responsive advice in order to facilitate effective behavioural change in diabetes management. $\mathrm{Wu}$ and colleagues ${ }^{3}$ also suggested that the most benefit was derived from intensive support which was based on an underpinning psychological approach following a MI style. This approach is increasing in popularity in health behaviour change interventions. A systematic review suggested that more research into MI applied to health behaviour change is urgently required ${ }^{4}$ although recent studies have shown the MI approach to be feasible in daily practice by healthcare professionals for diabetes management. ${ }^{5} \mathrm{Wu}$ and colleagues found that the most effective intensive telephone support follow-up was delivered by a specialist diabetes nurse rather than a trained healthcare professional or general nurse. This reduces the potential for conflicting advice, particularly in more complex cases, and may service to increase patient's confidence in advice and support since specialist diabetes knowledge has been identified as crucial to good quality care. ${ }^{6}$ The authors of this study also recognise the potential cost implications of specialist support and appropriately advocate an algorithm to determine the optimal mix of specialist and support staff in telecare models.

\section{Commentary}

Telephone consultation has been used to augment patient support services for T2DM, often with the intention of increasing participant knowledge and understanding of diabetes and individual self-care elements (eg, monitoring blood glucose levels, dietary behaviours, exercise and medication adherence) to promote self-management. More specifically, studies have shown that telephonebased support offered as an adjunct to behaviour modification interventions and in encouraging patient self-care strategies can show lasting positive effects in T2DM patients. ${ }^{7}$ This is significant given the rapid increase in the prevalence of diabetes worldwide. In response to this increasing demand, the efficiency and quality of services for people with diabetes remains at the forefront of public health strategy. This review demonstrates that telephone consultation or follow-up may offer some benefit within telecare models for the management of T2DM, if advice is appropriately targeted and tailored with trained healthcare 
professionals or diabetes specialists particularly in more complex cases. More research is needed into the mode of delivery. As the authors have themselves recognised the importance of the underpinning model in diabetes care compared with the mode of delivery, it seems prudent to recognise the potential for similar tailored intervention delivery to support diabetes care by other methods of telemedicine, such as internet, email or mobile phone text messaging. Inp articular, cellular phone subscription continues to grow at a faster rate than fixed telephones in developing as well as developed countries, providing a new rapidly advancing method to support clinical care worldwide.

\section{Competing interests None.}

\section{References}

1. Wu L, Forbes A, While A. Patients' experience of a telephone booster intervention to support weight management in type 2 diabetes and its acceptability. J Telemed Telecare 2010;16:221-3.

2. Piette JD. Interactive behavior change technology to support diabetes selfmanagement: where do we stand? Diabetes Care 2007;30:2425-32.

3. Gambling T, Long A. Tailoring advice and optimizing response: a case study of a telephone-based support for patients with type 2 diabetes. Fam Pract 2010;27:179-85.

4. Knight KM, McGowan L, Dickens C, et al. A systematic review of motivational interviewing in physical health care settings. Br J Health Psychol 2006;11(Pt 2):319-32.

5. van Eijk-Hustings YJ, Daemen L, Schaper NC, et al. Implementation of Motivational Interviewing in a diabetes care management initiative in the Netherlands. Patient Educ Couns. Published Online First: 16 July 2010. doi:10.1016/j.pec.2010.06.016. 
6. Stenner KL, Courtenay M, Carey N. Consultations between nurse prescribers and patients with diabetes in primary care: A qualitative study of patient views. Int J Nurs Stud 2011;48:37-46.

7. De Greef KP, Deforche BI, Ruige JB, et al. The effects of a pedometer-based behavioral modification program with telephone support on physical activity and sedentary behaviour in type 2 diabetes patients. Patient Educ Couns. Published Online First: 21 August 2010. doi:10.1016/j.pec.2010.07.010. 